\title{
Future Military Mobile Radio Communication Systems from Electronic Warfare Perspective
}

\author{
Harri Saarnisaari, Timo Bräysy \\ Centre for Wireless Communications \\ University of Oulu \\ Oulu, Finland
}

\begin{abstract}
Detection devices are becoming more wideband, faster scanning and their signal processing capabilities are increasing. In addition, sophisticated jamming devices have become rather powerful. As a consequence, threats to military radio communications have increased such that it is about time to discuss what features future military radio systems should have in order to battle against these increased menaces. This paper fills this gap by considering this problem from the point of view of electronic warfare physics. The result is a set of features that would help in the engagement. Furthermore, it discusses what effects these features have for radio system design. The proposed features add new properties, compared with legacy radios, that require new control functionalities that have to cooperate with old ones as well as together and also add radio hardware. Therefore, corresponding research problems will not be easily solved.
\end{abstract}

\section{INTRODUCTION}

Since the beginning of using radio communications in military operations opponents have tried to detect, classify and locate the transmitters and deny radio communications by jamming. As a consequence, the communicator has developed countermeasures to prevent these hostile actions which has led to new detection and denying strategies and so on. This game that can be called electromagnetic battle (EMB) on radio communications is still going on. Recent developments on radio fronts ends used in commercial software defined radio platforms that allow listening more and more spectrum with lower price tags as well as progress on signal processing capabilities that allow more real time computing means that detection devices have become rather powerful. This means increased detection and classification threat. At the jamming side the development is not so obvious and devices seem to be expensive though most sophisticated ones have become more effective and powerful. Although civilian mobile radio systems have developed recently a lot, military communication systems remain old fashioned. One reason is their long life cycle and another the cost of acquiring new systems by large numbers. Indeed, a lot of old fashioned legacy systems are seen in the field. Furthermore, new systems hold some of the sins of the legacy system. These sins are that they are limited to a certain frequency range, they have just a few if not just one possible transmit power levels and just a few data rates where modulation order and channel coding are used to adjust the behavior of the radio and radio network. Since the flexibility is limited these systems tend to have limited countermeasures possibilities. It is not easy to get all the facts from open sources to support these claims. Instead, many web-pages just claim (maximum) output power and frequency range. However, it is believed that the handheld ST@RMILLE radio by Thales ${ }^{1}$ provides a typical example of modern design. It has a fixed frequency band and bandwidth, $0 \mathrm{dBm}, 24 \mathrm{dBm}$ and $33 \mathrm{dBm}$ output power levels and a fixed data rate. It does not seem to have spread spectrum properties. Another example is Harris adaptive networking wideband waveform, but just little information can be found from open sources [1]. However, the modern radio devices may support several waveforms and using them smartly increases robustness, as might be the case in this Harris Falcon III AN/PRC-152A radio $^{2}$

This paper takes the physical limits of communications, detection and jamming into account and discusses the desired features of the future mobile military radio communication systems from that perspective. This is important since detection systems are approaching the limits and radio systems should react accordingly. The scientific community has not yet started discussing these, at least openly, and it is important to open the door as in this paper since these complicated problems, as will be seen, require a lot of research. Often one research effort makes certain improvements such that many research efforts are required until sufficient solutions are ready. Therefore, open international research is a key for good products. As said, this seems to be the first open paper that discuss required features from the EW perspective. The viewpoint is that of the communication system. Detection and jamming system designers should make their own conclusions. The limits are first discussed. Then, more importantly, the required features based on the limits to the radio systems are discussed along with the problems they set for radio systems. The results of these discussions show what directions should be taken in the future if real electronic warfare (EW) capable radio systems will be planned. However, solutions to

\footnotetext{
${ }^{1}$ http://www.ste.com.my/products-starmille.html

${ }^{2}$ https://www.harris.com/sites/default/files/downloads/solutions/harrisfalcon-iii-an-prc-152a-wideband-networking-handheld-radio.pdf
} 
the problems are not given but they are left also for research colleagues.

\section{EW PHYSICS ON BACKGROUND}

For simplicity the free space propagation is used in calculations below and throughout the paper. However, similar conclusions hold for more practical models that should be used for lower antenna heights, long distances etc. even though the values would be different. Furthermore, as in the free space model where the propagation attenuation is inversely proportional to the square of the carrier frequency, also in other models signals at higher frequencies attenuate more. This means that with the same transmit powers the effective ranges would be shorter at high frequencies than at low frequencies.

\section{Communications}

Certain data rate in communications requires certain signalto-noise ratio (SNR) or, more generally, in interfered environments certain signal-to-interference-plus-noise ratio (SINR) to operate properly. The transmit power (including antenna effects both in the transmitter and receiver in this paper) then determines that achievable communications range. Let $P_{r}$ be the received signal power, $N_{0}$ the noise density of the receiver including noise figure, $B$ the bandwidth (about the channel symbol rate) and $J$ the interference (jamming) power on the receiver. Then

$$
\mathrm{SINR}=\frac{P_{r}}{N_{0} B+J}=\frac{1}{1 / \mathrm{SNR}+\mathrm{JS}}
$$

where $\mathrm{JS}=J / P_{r}$ denotes the interference-to-signal power ratio. Often SNR $>>0$ such that in interfered environments $\mathrm{SINR} \approx 1 / \mathrm{JS}$ such that interference becomes a limiting factor. Note that herein the hit rate, i.e., what portion of the signal is hit by interference, is not taken into account (interested readers may refer to [2]). The required SINR depends on the modulation order and coding. For example, a typical half rate convolutional coding with soft decoding requires channel bit error rate (BER) $10^{-3}$ for data transmission (approximately). For binary phase shift keying (BPSK), 16-ary quadrature amplitude modulation (16QAM) and 256QAM these corresponds $6 \mathrm{~dB}, 12 \mathrm{~dB}$ and $19 \mathrm{~dB}$ SNR, respectively [3]. In addition, since $\mathrm{SNR}=P_{r} / N_{0} B$ it is obvious that increased bandwidth means decreased SNR, i.e., increased data rate without power compensation means shorter communication ranges.

In principle, if the communication system wishes to hide it should use minimum transmit power to achieve the required SNR level at the receiver. However, this would leave the receiver very sensitive to interference such that often some SNR margin is used, i.e., a target SNR value is a minimum SNR value increased by a margin.

\section{Detection}

The typical detector is the energy detector. More specifically, due to platform development a channelized energy detector based on the fast Fourier transform (FFT). Often detectors scan a certain wider frequency band with an instantaneous bandwidth. Though there are exact detection performance results available [4] rules of thumb are used herein for simplicity. First, at low input SNR region the output SNR of the energy detector is $\mathrm{SNR}_{o}=\left(\mathrm{SNR}_{i}\right)^{2} T W$, where $T$ denotes the integration time and $W$ the input bandwidth [5]. At high input $\mathrm{SNR}$ it is linear relation $\mathrm{SNR}_{o}=\mathrm{SNR}_{i} T W$ [5]. In a FFT based detector each frequency bin or cell has $T W=1$. In order to detect weaker signals several FFT outputs (spectrum estimates) have to be added together but that slows scanning the frequency range. As a consequence, there are design tradeoffs.

It is often argued that direct sequence (DS) signals are hard to detect. DS signals have a wider bandwidth than the data rate implicates. Therefore, the name spread spectrum techniques. The amount of spreading is called processing gain (PG). However, the transmit power is kept the same. A consequence of this spreading is that in the narrowband detector only a fraction of signal power $P$ is received, i.e., $\mathrm{SNR}_{i}=P / P G /\left(N_{0} B\right)$, whereas in the wideband detector the power is captured totally but the noise power increases due to increased bandwidth, i.e. $\mathrm{SNR}_{i}=P /\left(N_{0} B \times P G\right)$. As a consequence, both the narrowband and wideband detector are equally sensitive against DS signals assuming that the integration times are equal. In practice, though, the transmit power of communication devices is often so high that there is enough power to detect even DS signals without additional integration time, as will be seen in the numerical examples. If the bandwidth extension is used for increased data rate then the same phenomena occurs. Naturally, in this case, if the communication system increases its transmit power to compensate for reduced range its detectability is closer to the narrowband reference.

Sometimes radio system intelligence reveals that communication signal has a certain fixed preamble or cyclic structure, like in the orthogonal frequency-division multiplexing (OFDM). This would allow the detector to use (nearly) optimum feature based power detection and classifier [4] and make the signal rather vulnerable without excessive collection of samples. For example, the communications preambles are typically designed such that preamble detection is a tad more sensitive than reliable data detection, i.e., signal detection range would be (somewhat) longer than that of the communication range. If the preamble would be known to the detector, the detector could use this for its advantage albeit matched filtering with large frequency uncertainty is computationally a demanding problem limiting the usability of this idea.

\section{Jamming}

Using the free space propagation model

$$
J S=\frac{P_{J T}}{P_{T}}\left(\frac{d_{c}}{d_{j}}\right)^{2}=\frac{P_{J T}}{P_{T} \alpha^{2}},
$$

where $\alpha=d_{j} / d_{c}, P_{J T}$ and $P_{T}$ denote the transmit jamming and communication power, respectively, and $d_{j}$ and $d_{c}$ denote the jamming and communication distance, respectively. Obviously this does not depend on the operation frequency but just 
the jamming and communications transmit powers (if antenna effects are integrated to them) and relative distance.

While frequency hopping $(\mathrm{FH})$ spread spectrum technique is about avoiding hits by a jamming signal (and a detector scan) DS technique is about reducing jamming effect by PG. For example, $\mathrm{PG}=100$ means $20 \mathrm{~dB}$ improved jamming properties but often the situation may be such that even this does not help a lot since JS is so large.

If the Journal of Electronic Defence (JED) and its list of jamming devices (like in June 2016 issue) and their power levels are browsed through, it can be observed that jamming powers are often at $100 \mathrm{~W}$ class in lower frequencies and $10 \mathrm{~W}$ class at higher frequencies (there is a lot of variation). These numbers are good references when numerical results are viewed.

Jamming is often against a certain target since it is not desired to jam own signals. Therefore, often a prerequisite for starting jamming is that the target is detected and classified. In that case the jammer could concentrate all its power to the target signal bandwidth and be as efficient as possible. This is called a follower jammer mode. Alternative is a wideband or barrage jammer mode where a wider bandwidth, e.g. the expected operation bandwidth of the target system, is jammed. However, this means that jamming power to the instantaneous signal bandwidth is greatly reduced.

\section{NUMERICAL EXAMPLES}

To get some insights what the presented physics means in reality, a set of results are shown using the free space propagation model. Since, in practice, radio horizon limits the ranges but the free space propagation model does not, the analysis is limited to $40 \mathrm{~km}$ although higher altitude platforms may be operable from further off.

The target signals are $25 \mathrm{kHz}$ and $1 \mathrm{MHz}$ signals $(B)$. The latter could be understood either a higher data rate signal or a DS signal. Instead of using actual detector models, a simpler approach is selected. It is assumed $25 \mathrm{kHz}$ cell size in a channelized detector. For $T W=1$ about $16 \mathrm{~dB} \mathrm{SNR}_{i}$ is required for reliable detection (in white Gaussian noise channels) [4]. In addition to this also $\mathrm{SNR}_{i}=0 \mathrm{~dB}$ is used to show what about 40 times integration, and thus reduced scanning speed, could do. For the used wideband signal its power at $25 \mathrm{kHz}$ band is considered.

\section{Communication vs Detection Range}

The first set of results in Figs. 1 to 4 shows the maximum communication and detection range as a function of the transmit communication power. The aim is to show at which power communications and detection are possible and are there differences. In addition to above mentioned detectability estimates communication ranges are shown for the minimum SNR (for given modulation) and the target SNR when the SNR margin is $10 \mathrm{~dB}$. Also a feature detector is modeled such that it is 32 data symbols long and has to have $16 \mathrm{~dB}$ SNR at the detector (matched filter) output.

The results show that hiding from detection using traditional a few watts output power levels is difficult, if not impossible

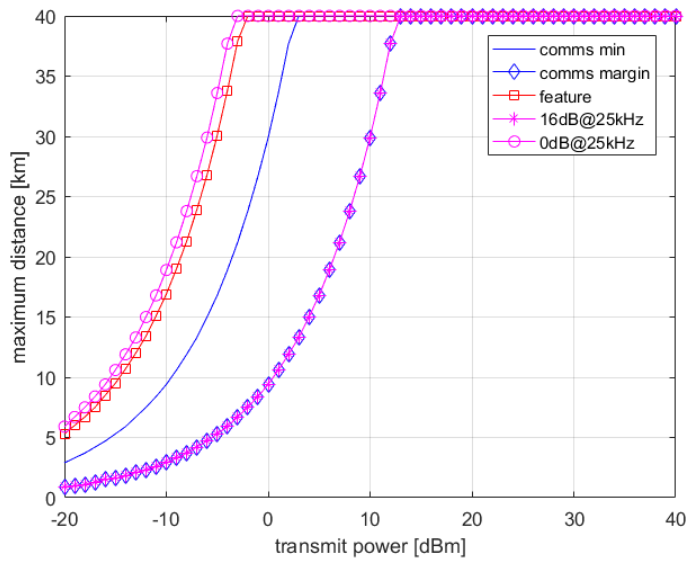

Fig. 1. The maximum communication and detection range as a function of the transmit communication power for BPSK, $400 \mathrm{MHz}$ carrier, $25 \mathrm{kHz}$ signal.

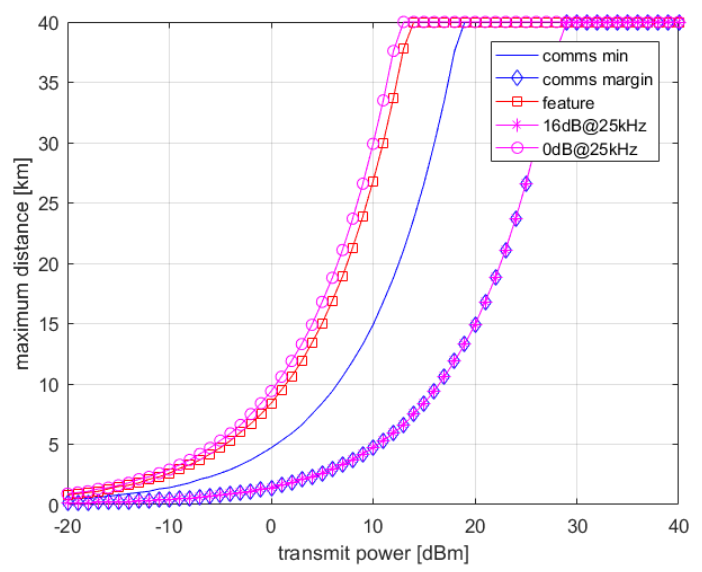

Fig. 2. The maximum communication and detection range as a function of the transmit communication power for BPSK, $400 \mathrm{MHz}$ carrier, $1 \mathrm{MHz}$ signal.

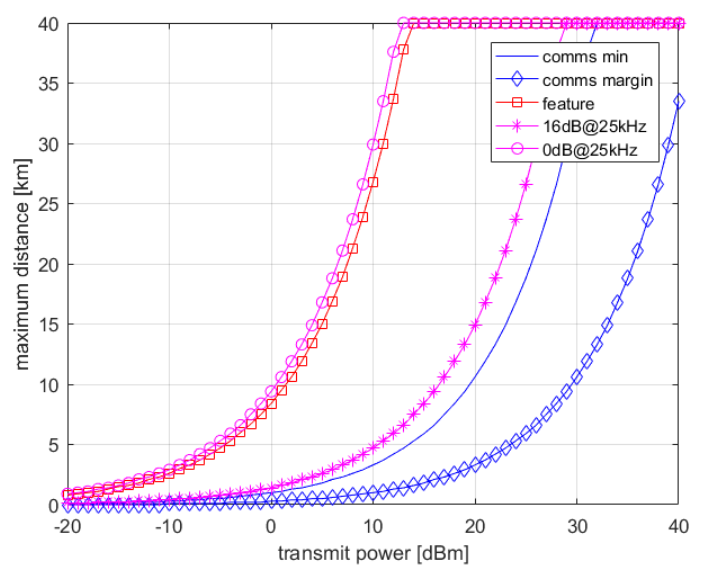

Fig. 3. The maximum communication and detection range as a function of the transmit communication power for 256QAM, $400 \mathrm{MHz}$ carrier, $1 \mathrm{MHz}$ signal. 


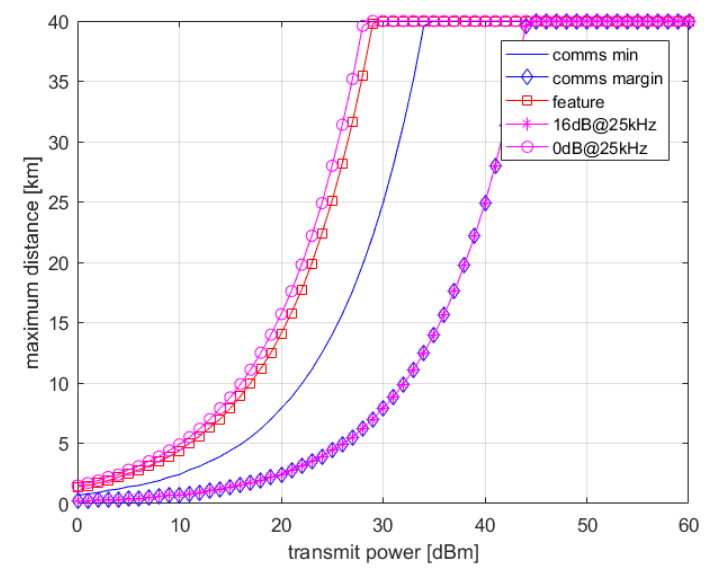

Fig. 4. The maximum communication and detection range as a function of the transmit communication power for BPSK, $2.4 \mathrm{GHz}$ carrier, $1 \mathrm{MHz}$ signal.

at low frequencies since the detection ranges are very long. At higher frequencies the detection range is reduced. Another observation is that the SNR margin makes the communication system more vulnerable to detection since even fast scanning (one shot) detector is often sufficient. Higher modulation orders increase this vulnerability dramatically. Detectability of a DS signal with respect to a corresponding narrowband signal could be seen by comparing Fig. 1 with Fig. 2. If the latter is a DS signal it would have $\mathrm{PG}=40$ or $16 \mathrm{~dB}$. But still, the DS signal is fast detected from long distances if usual $1 \mathrm{~W}$ or higher transmit powers are used.

One should try to make detection range so short that it is practically impossible, i.e., the detector must become too close. What is short enough depends on your requirements. However, there are countermeasures the observer may use. The observer may oppose this using flying detectors that may become very close or may have better signal propagation conditions. Or it may use directional antennae that shift it to right in the result curves, i.e., to the direction of longer detection distances.

Techniques for making short detection ranges are listed here:

1) Use as low power as possible, but this reduces communications range, possibly data rate or both.

2) Use the DS technique since it limits detectability.

3) Use higher frequencies but this reduces communications range, possibly data rate or both.

4) Use a directional antenna and hope that the detector is on its sidelobe. This means that the communicator stays at the desired power level in the curves whereas the detector is moved to left, to direction of reduced detection range. The shift in $\mathrm{x}$-axis is equal to difference between sidelobe and main lobe gain (in $\mathrm{dB}$ ).

Then there are tricks that do not really make the range shorter but instead make detection more difficult.

1) Do not expose preambles (worst) and cyclic features (less worse) to the opponent since that prevents using fast and sensitive feature detectors.

2) Force the detector to use fast scanning (that is least sensitive) or even hide from a scanning detection using

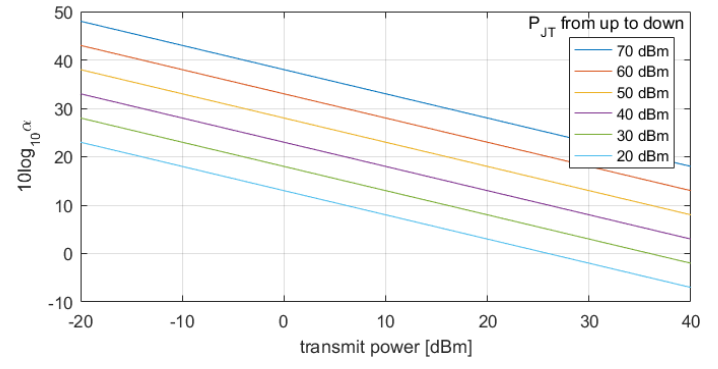

Fig. 5. The jamming-to-communication link range ratio $\alpha$ as a function of transmit communication power for different jamming power values and BPSK signal.

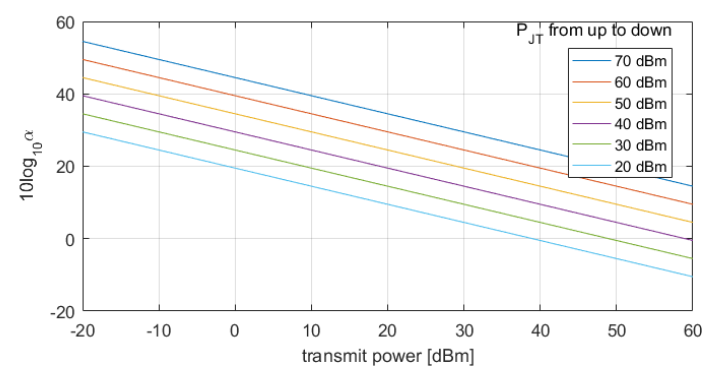

Fig. 6. The jamming-to-communication link range ratio $\alpha$ as a function of transmit communication power for different jamming power values and 256QAM signal.

the FH technique or even frequency band hopping.

3) Apply radio silence or listening only practices, or use cables.

\section{Communication vs Jamming Range}

The second set of results in Figs. 5 and 6 shows what the jamming-to-communication link range ratio ( $\alpha$ from (2)) must at least be as a function of transmit communication power for different jamming power values. Read the result curves so that jamming fails if $\alpha$ is larger than the line you are looking at. This indeed shows how far the jammer must be with respect to the target for a given communication range. More precisely, if the ratio is less than one, the jammer must be closer the target than the transmitter of the communication link.

It is obvious from the results that with typical jamming and communication powers $\alpha>>1$ (10-100) meaning that the jamming range is much longer than the communication range. If the communication transmit power is reduced, e.g. for hiding, the communication system can be jammed very far away (compared its range). As in detection, one should force jamming range to such a short value that it becomes impossible for given situation. Note that if effective jamming power is reduced by $20 \mathrm{~dB}$ the effective jamming distance is reduced by factor ten.

There are a few possibilities for reducing jamming effects. Except the first one the methods reduce effective jamming power to the signal, i.e., jamming power to be considered goes towards lower lines in the result figures for a given communications transmit power.

1) Use as high transmit power as possible. 
2) Use directional antennae.

3) Use the DS technique.

4) Force the jammer for the wideband mode instead of the follower mode where all power could be concentrated to the signal. This means that detection and classification should be prevented.

5) Use low modulation orders since they tolerate more jamming power.

6) Use short hops since they shorten the jamming range by the same reduction factor (in equal propagation environments).

In addition, certain signal processing methods married with the DS technique may mitigate some jamming signals and digital beamforming antennas may produce nulls against interference and are very effective [6]. However, the latter are very expensive and have a large size at lower frequencies. Cables are also good against radio jamming, but they have their own problems.

\section{Consequences to Future Radio Systems}

This section discusses what are the consequences of above observations for the military radio systems and how these affect the system design and should be taken into account when novel systems will be designed. These consequences are listed as desired features of radio systems. Many required features yield to similar consequences in the system design.

\section{Power Control}

Low powers for hiding and high ones for anti jam (AJ) properties; low powers for short distances and high powers for longer ones; low powers for low modulation orders and high ones for higher orders. As a consequence, there are a lot of trade-offs when power control is dealt with. Furthermore, it also affects networking functionalities.

Power levels that make detection distances (too) short for the opponent also make communication ranges short. This mean that network topology is different at low powers than it is with usual powers. This means that network initialization, joining and operation may change since the links are shorter and there are more multihop routes. At least this means that corresponding functionalities at the networking and medium access layers should be flexible enough to handle both situations marvellously. If dead radio links by jamming and listen only mode (which, basically, is an informed one-way broken link) are taken into account, the upper layers must be very clever.

The power levels should, according to the example results, go from negative tens of $\mathrm{dBm}$ for hiding to several watts or as high as possible for AJ properties.

\section{Modulation (and coding) Control}

There is a tad over $10 \mathrm{~dB}$ difference on SNR requirement between BPSK and 256QAM modulations. Coding could make a few $\mathrm{dB}$ additional difference. Taking these benefits into account may make the difference between being detected/jammed or not. Naturally, this limits the throughput of the network, and especially so if the power control is included too. Indeed, low data rates may mean that some applications (services) cannot be used or that information they could provide is limited. Therefore, adaptive applications are required and this may have effects to the user behavior as well.

Low data rates may be bottleneck for some medium access, routing and networking algorithms, i.e, the ones used/proposed now do not operate anymore if these low data rate modes are accepted or they may choke the whole network such that payload cannot be transmitted if applied for such modes.

\section{DS Control}

Moderate ten to hundred times spreading adds 10 to 20 $\mathrm{dB}$ against both detection and jamming. But as seen from the numerical results this does not help alone but should be adopted with power and modulation control; the DS technique it is not a game changer as such.

The DS technique reduces the data rate by PG and that may have been too high sacrifice for increased EW properties such that the DS technique is quite seldom used. It could be said that the DS technique wastes the given spectrum by PG and since spectrum is scarce the DS technique is not so popular. But when the threats increase and become more dangerous this penalty may have to be paid.

The lowered data rate cause the same effects than readily discussed above. However, there are other consequences too. The synchronization preamble has to be adapted to include spreading. This means that the synchronization preamble would be different for the DS mode and the non-DS mode. In addition, synchronization algorithms should be modified accordingly though the principles would be the same. If just one preamble structure would be used, the DS mode preamble should be used and this would mean waste of symbols in the non-DS mode, i.e. reduced data rate, which would be especially harmful in short packet systems. The radio systems should (automatically) be able to handle both situations.

\section{Networking}

The power, modulation and DS control affect networking functionalities as readily discussed. The short links in the low probability of detection (LPD) mode mean short links and multiple hops to reach a distant destination node. In the AJ mode links could, in principle, be longer since the transmit power is high but the system should favour short links. This may make networking management an issue, which is enhanced by a fact that a part of the network could be in the LPD mode and another part in the AJ mode.

Since the data rate may be throttled into a minimum under stress the medium access, routing, topology control and so on algorithms should be designed based on the minimum (minimal overhead) or they should be adaptive if needed for better efficiency at the high data rate (HDR) situations. An example about this is header compression that is a widely applied technique to reduce overhead [7]. Data prioritization is also most probably needed as well as application adaptability.

But the networking functions can do more. They could route traffic to avoid areas that are known to have harmful detectors or jammers. And, if directional antennae are used, they can 
arrange topology such that detectors and jammers are not at the main beam [8].

The chaos is added by the required frequency variability to be discussed next and that has to be handled in the network management level too, e.g., by means developed within what is usually called cognitive military radios.

\section{FH Control}

It is easy to detect a strong enough signal and if it is in a fixed frequency, it will be easily jammed by a follower jammer. If the target changes its frequency for another fixed frequency, the jammer easily follows. Fast enough FH makes detectors/jammers life more miserable since it tries to avoid a scanning detector and limit the hit time a follower jammer can have after it has detected the target signal and generated a jamming signal that still has to propagate to the target. At worst, the detector is forced for fast scanning that is not so sensitive against weak signals and the jammer is forced to wideband jamming that reduces effective jamming power.

A drawback is complicated signal structure since FH synchronization is required too. In addition, radio devices tend to become more costly, bulky and power hungry. Frequency management may become more complex since dynamic spectrum usage algorithms should take hopping patterns into account.

\section{Band Hopping}

A step further from frequency hopping is to use different frequency bands in different situations, considered, e.g., here [9]. Escaping of jamming to totally another frequency band may cause a lot of confusion to the opponent. But band hopping has another purposes too.

If the radio system is capable on operating at two simultaneous frequency bands it could select which band to use at the moment or even use band diversity at least for critical messages. This would greatly enhance routing diversity possibilities (if such are applied).

Furthermore, since the signal attenuates more on higher frequencies these higher bands may be favored in the hiding mode. But since this affects also the communication range and, consequently, topology, the networking functionalities should behave correspondingly, as discussed above.

Drawbacks are increased control complexity since frequency band control has to be included, and its consequences to radio devices. However, very wideband software defined radio devices are readily available for research purposes and probably those will appear soon for military markets with sufficient protection against radio frequency (RF) weapons.

\section{Directional Antenna}

If a detector or a jammer is in the sidelobe direction of a sectoral antenna their performance will be reduced. In digital beamforming antennae a null could be directed towards the opponent. Sectorization or beamforming mean that networking functions should be modified such that the main beam will be searched when connections are established, i.e., angular synchronization is required in addition to others. How many beams can be listened simultaneously affects the (average) speed of synchronization. Especially at low frequencies directional antennae tend to be bulky structures (due to physics) that may limit their usage.

\section{Listen Only Mode}

Radios that are in the listen only mode cannot be detected and located but messages they are intended to receive can. Therefore, also these nodes are susceptible to jamming. This mode affects the radio topology and, as a consequence, networking functionalities.

\section{A. Further Features}

Then there are other features that do not directly stem from the shown numerical results but are closely related since they operate those features.

\section{Cognition}

Cognition is a wide topic since there are many aspects that could be covered. Anyway, the target is clear. The cognitive management systems should be designed such that the available resources are used the best way given the situation at hand. But the task is not an easy one as can be observed from the previous discussion in this section; there are numerous moving parts from the EW point of view. In addition to these there are more familiar aspects like dynamic spectrum management typical in cognitive radio systems under research and development.

The EW viewpoint means also an additional cognition component. In principle, it is about learning from own, detector's and jammer's reactions to different stimulus such that opponent's life is made miserably. A game theoretic analysis is shown in [10]. The radio should learn how a jammer reacts its signals in hit, evasive action, hit, ... chain and adjust own behavior correspondingly. If the reactions are always the same but successful, then own evasive action should be changed. The radio may have a bunch of evasive action plans to choose from and it should learn which one to select for certain cases. The radio should recognize if small disturbances are actually from a jammer; evasive actions should not be started and possibly exposed if not needed. This may occur especially in a low power mode with small SNR margins since even small interference cause disturbances and in any case since there might be internal interference due to poor or failed frequency management.

\section{System Programmability}

Once the tricks of the radio system have been used and the opponent has developed successful countermeasures for all of them the behavior of the system should be changed such that communication would again be possible under jamming. This might come ones way in a prolonged conflict. This requires system programmability, either externally or futuristically as a result of self-learning and corresponding reprogramming. An example of this could be reprogramming of evasive actions. Naturally, this requires that the radio system is not a black box that just does its job but a programmable unit. 


\section{Transformable Waveform}

Once the communication signal is detected, if not readily known, its observed characters are saved into the opponent's library of threat signals. A novel signal is a surprise and may cause uncertainty into opponent's reactions. Instead of buying new radios the old ones could have transformable waveforms that can change their characters sufficiently. This would add new control layer to ever increasing pile of layers or degrees-of-freedom. In principle, it could be possible to mimic opponent's signals and cause even more uncertainty. Naturally, this would cause uncertainty also for own signal detection and classification tasks such that properly working electromagnetic battle management (EMBM) system would be needed to tell who is who.

Acquisition announcements as well as publicity requirements often tell what radio devices are bought and used. Intelligence people can easily collect all public (and maybe some non-public) information for their libraries. However, it is essential to buy or produce radios that have secrets, or that can be programmed to have them. Only that way the opponent can be surprised.

\section{Discussion}

This paper has discussed desired military radio properties from the EW viewpoint. Obviously these desired features mean that systems would become more and more complex. The radio devices should be wideband or multiband and transmit power range should be wide or at least have several small, medium and large values. Directional antennae should be used whenever possible. These could be digital beamforming antennae, which mean more flexibility in the network design, or sectoral antennae that either listen all the sectors simultaneously or could very fast hop from a sector to another. However, maybe the biggest challenge is to develop properly working cognitive control algorithms to guide radio's behavior. As seen in the list of desired features, there are many things that have to be taken into account and many degrees-offreedom such that the task is not by any means a simple one. Also co-operation between the modes is an open problem. By this it is meant that different parts of the network may be in a different mode, e.g., one unit wants to hide (from radio detection) and another is under jamming or using regular mode. Or, completely different waveforms are used in different parts of the network. The system must be build such that this is possible. A summary of the main observations and concerns that paper has raised is shown in Fig. 7.

Most of the shown features are additional to those discussed in the literature, especially concerning cognitive military radios. This means that these should be build in addition to those readily under development like dynamic spectrum management whose major goal has been putting own radios operation in the same area to work together without interfering each other, e.g., [11]. The minor goal, if it has been the goal at all, has been the development of efficient cognitive AJ properties.

It is reminded that most discussed features are not by any means novel. However, they are presented here in one place as a result of analyzing EW effects. This brings out their importance from another than usual perspective since this has not been done previously. Especially the importance of power control, short hops and directional antennae were highlighted since they have positive effects of the LPD and AJ properties.

\section{REFERENCES}

[1] C. Martin, "Progress in tactical wideband waveform development," Battlespace, vol. 13, 2011.

[2] H. Saarnisaari, "Sweep jamming hit rate analysis for frequency agile communications," in International Conference on Military Communications and Information Systems (ICMCIS), 2016.

[3] J. G. Proakis, Digital Communications, 3rd ed. New York: McGrawHill, 1995.

[4] S. Kay, Fundamentals of Statistical Signal Processing: Detection The ory. New Jersey, USA: Prentice Hall, 1998.

[5] H. Klipper, "Sensitivity of crystal video receivers with RF preamplification," The Microwave Journal, vol. 8, no. 8, pp. 85-91, August 1965.

[6] H. Saarnisaari, H. Puska, and P. Lilja, "Interference mitigation techniques for single and multi antenna receivers," in The first European Conference on Antennas and Propagation (EUCAP), 2006.

[7] B. n. Cheng, J. Wheeler, and B. Hung, "Internet protocol header compression technology and its applicability on the tactical edge," IEEE Communications Magazine, vol. 51, no. 10, pp. 58-65, October 2013.

[8] H. A. Mustafa, X. Zhang, Z. Liu, W. Xu, and A. Perrig, "Short paper: Jamming-resilient multipath routing leveraging availability-based correlation," in Proceedings of the Fourth ACM Conference on Wireless Network Security, ser. WiSec '11. New York, NY, USA: ACM, 2011, pp. 41-46. [Online]. Available: http://doi.acm.org/10.1145/1998412.1998421

[9] J. Xu and C. Yu, "Improving network energy efficiency through cooperative idling in the multi-cell systems," EURASIP Journal on Wireless Communications and Networking, no. 165, 2011.

[10] W. G. Conley and A. J. Miller, "Cognitive jamming game for dynamically countering ad hoc cognitive radio networks," in MILCOM 2013. 2013 IEEE Military Communications Conference, Nov 2013, pp. 11761182.

[11] L. Rose, R. Massin, L. Vijaydran, M. Debbah, and C. Le Martret, "CORASMA program on cognitive radio for tactical networks: High fidelity simulator and first results on dynamic frequency allocation," in Proceedings of the IEEE Military Communications Conference, 2013. 

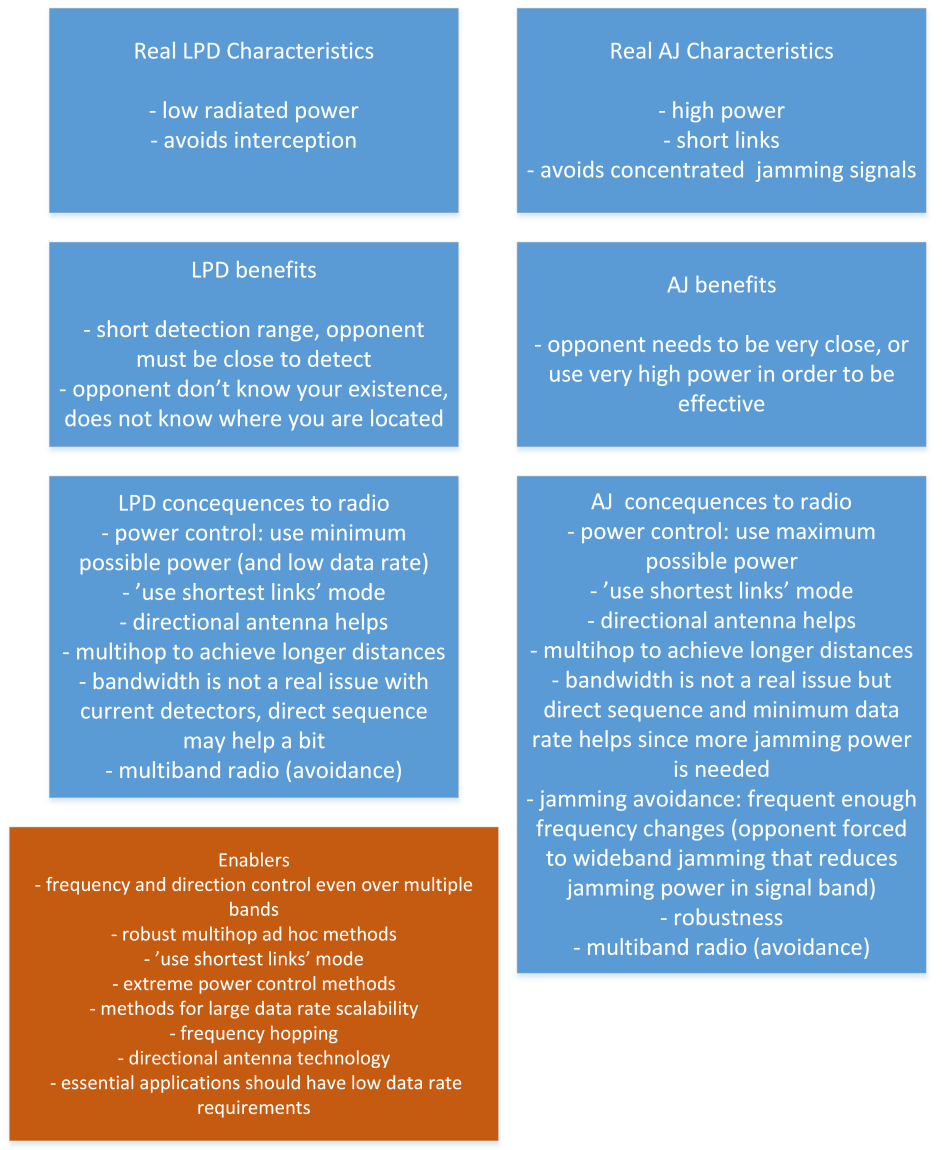

Fig. 7. A summary of the discussed properties and concerns.

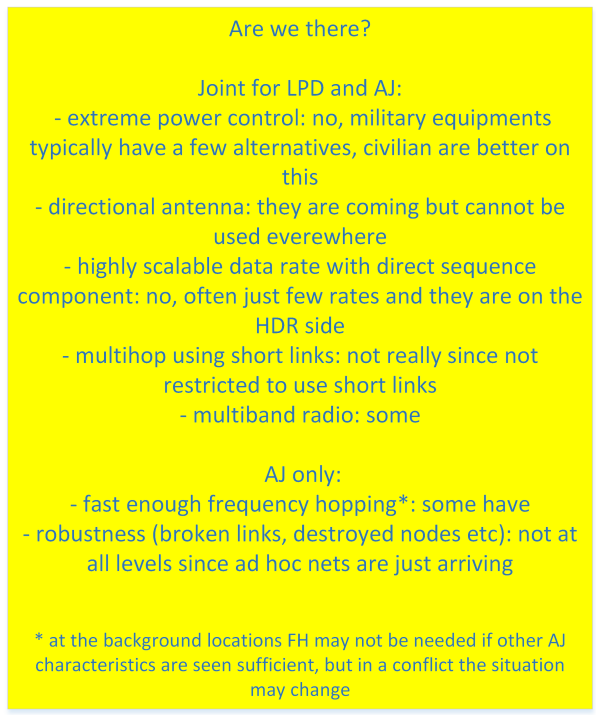

Naturally waveforms should not have weak points where opponent can attact or utilize like:

- known synchronization sequence

-known hopping pattern or too slow hopping

- predictable behavior after jamming (e.g. always goes to a certain frequency)

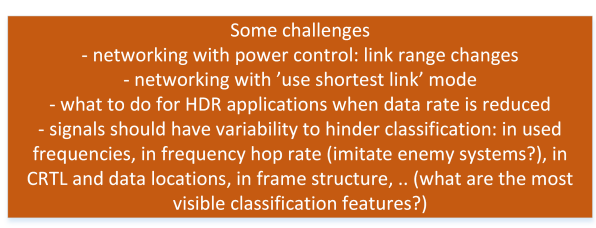

